\title{
Current Surveys of the Seroprevalence of Borrelia burgdorferi, Ehrlichia canis, Anaplasma phagocytophilum, Leishmania infantum, Babesia canis, Angiostrongylus vasorum and Dirofilaria immitis in Dogs in Bulgaria
}

Nikola Pantchev' $(\bowtie)$, Manuela Schnyder², Majda Globokar Vrhovec', Roland Schaper ${ }^{3}$, Ilia Tsachev ${ }^{4}$

${ }^{1}$ IDEXX Laboratories, 71636 Ludwigsburg, Germany

${ }^{2}$ Institute of Parasitology, Vetsuisse Faculty, University of Zurich, 8057 Zurich, Switzerland

${ }^{3}$ Bayer Animal Health GmbH, 51368 Leverkusen, Germany

${ }^{4}$ Department of Microbiology, Infectious and Parasitic Diseases, Faculty of Veterinary Medicine, Trakia University, 6000 Stara Zagora, Bulgaria

Corresponding author:

Nikola Pantchev

$\triangle$ E-mail: nikola-pantchev@idexx.com

\begin{abstract}
Canine vector-borne diseases (CVBDs) have increasingly become a focus of interest in recent years. Some of the CVBDs are zoonotic and may therefore also represent a risk for the human population. Different factors are in discussion to explain the expansion of vectors and pathogens into formerly unaffected areas. Knowledge of the prevalence and distribution of CVBDs in Bulgaria is scant overall and most data rely on single case descriptions. The aim of the present study was to determine the seroprevalence of important CVBDs in 167 dogs from central-southern Bulgaria (Stara Zagora), with special emphasis on hitherto
\end{abstract}

uninvestigated babesiosis and angiostrongylosis, on poorly investigated Lyme borreliosis and canine granulocytic anaplasmosis, and on the potentially zoonotic dirofilariosis and leishmaniosis. Relatively high prevalence rates were documented for anti-Babesia canis antibodies, Dirofilaria immitis antigen (16.2\%; 27/167 each), anti-Ehrlichia canis (21\%; 35/167) and anti-Anaplasma phagocytophilum antibodies (30.5-46.1\%; 51-77/167), while Borrelia burgdorferi seroprevalence was low (2.4\%; 4/167). All samples were negative for Leishmania infantum antibodies and Angiostrongylus vasorum antigen and antibodies. In total, $64.7 \%$ (108/167) 
of the samples indicated infection or exposure to at least one agent and a high proportion of dual infections (39.8\%; 43/108) was demonstrated. Multiple infections with up to four different organisms were also detected. Our data underline the importance of CVBDs and especially of co-infections which could influence the clinical outcome in dogs.

\section{Introduction}

Canine vector-borne diseases (CVBDs) have increasingly become a focus of interest in recent years. Climate change and biotic factors such as an increase in (wild) reservoir abundance, changing habitat structure, socio-political changes and, especially for dogs, increased travelling and dog imports for welfare reasons are discussed in this context as potential factors in the expansion of vectors and pathogens into formerly unaffected areas. Some of the CVBDs are zoonotic and therefore may also represent a serious risk for the human population. Knowledge of the prevalence and distribution of CVBDs in Bulgaria is scant overall and most data rely on case descriptions. Furthermore, data about some agents, such as the protozoan Babesia spp. or the metastrongyloid nematode Angiostrongylus vasorum, are completely lacking. The situation in this country is even more complicated as stray dogs are still common and are being imported for welfare reasons into other countries (e.g. Germany). Stray dogs, in particular, are not protected by preventive measures against infestation with ticks, mosquitoes, sand flies or against the development of the filarial heartworm Dirofilaria immitis and therefore pose a potentially considerable reservoir for vector-transmitted infections. In Bulgaria, canine monocytic ehrlichiosis (CME) caused by Ehrlichia canis was first reported in 2003 (Tsachev 2006). Subsequently, a seroprevalence of $37.5 \%$ in the northern part of the country and $30 \%$ in southern Bulgaria was determined (Tsachev et al. 2006a, b). Furthermore, a confirmed clinical case of a coinfection of E.canis with Hepatozoon canis in a dog from Stara Zagora was documented (Tsachev et al. 2008a). Thereafter, several cases of CME were reported not only from Bulgaria but also from neighbouring Greece (Tsachev 2009; Tsachev and Petrov, 2009; Tsachev et al., 2013).

In contrast to E.canis, there are no published studies on the occurrence and prevalence of canine Babesia spp. from Bulgaria. This is surprising, because babesiosis is well known in dogs in this country (Tsachev, personal communication). Recent investigation of 295 dogs for ticks revealed an infestations rate of $23.7 \%$ for ticks, with $R h i$ picephalus sanguineus and Ixodes ricinus found in $19.7 \%$ and $6.4 \%$ of the dogs respectively, while obviously no Dermacentor reticulatus ticks were identified (Kirkova et al. 2013). These data were in part confirmed by another study investigating ticks $(\mathrm{n}=472)$ collected from humans $(\mathrm{n}=32)$, animals $(\mathrm{n}=264)$ and environment $(\mathrm{n}=176)$ in nine major districts in Bulgaria (Ivanov et al. 2011). Latter study revealed $R$. sanguineus in $31.4 \%$ (148/472) and I. ricinus in $25.4 \%$ (120/472) of the cases, but also D. reticulatus was found, even though at a lower rate $(3.6 \% ; 17 / 472)$. Unfortunately, latter study provides no information about the exact source of $D$. reticulatus ticks identified. These data point to possible transmission of Babesia vogeli in dogs in this country through $R$. sanguineus, but the presence of B. canis with D. reticulatus as a vector cannot be excluded.

There are only restricted data on CVBDs transmitted by I. ricinus in Bulgaria. A single clinical case of canine Lyme borreliosis (LB) has been reported so far (Popova and Kamenov 1998), as has one case of a co-infection with E. canis and Borrelia burgdorferi (Tsachev et al. 2007a). Subsequent studies demonstrated a low incidence of dogs with compatible clinical signs of LB $(0.8 \% ; 4 / 490)$, mainly in the south (Tsachev and Dimov 2012). Canine granulocytic anaplasmosis (CAG) caused by Anaplasma phagocytophilum which is also transmitted by Ixodes spp., was demonstrated for the first time in Bulgaria in 2008 (Tsachev et al. 2008b). Subsequent studies showed its occurrence only 
in southern regions (3.5\% in Plovdiv and $17.4 \%$ in Stara Zagora) and not in northern Bulgaria (Tsachev 2009).

The first cases of dirofilariosis in dogs in Bulgaria were documented in 1997-1999. Microfilariae determined as larval stages of Dirofilaria immitis were found in both pet (1.4\%) and stray dogs (12.5\%; Georgieva et al. 2001). In studies conducted in southern Bulgaria from 2001 to 2007 ( $\mathrm{n}=511$ dogs) microfilariae of $D$.immitis were detected in 47 (9.2\%) and of Dirofilaria repens in 7 (1.4\%) of the dogs tested (Kirkova et al. 2008). Some potential mosquito vectors for filarial nematodes were shown to occur in this country (Anopheles maculipennis, Anopheles cinereus, Culex pipiens, Culex perexiguus, Aedimorphus vexans and Coquillettidia richardii; Mikov 2011).

The first confirmed clinical cases of canine visceral leishmaniosis in Bulgaria in 2006 were located on the border with Greece (Tsachev 2009; Tsachev et al. 2010). Sporadic cases were also reported from other southern parts of the country (Tsachev 2009). Potential vectors for Leishmania sp. present in Bulgaria are Phlebotomus balcanicus, Ph.perniciosus, Ph.sergenti, Ph.papatasi and Ph.tobbi (Mikov and Harizanov 2012).

The aim of the present study was to determine the seroprevalence of important CVBDs in dogs in Bulgaria with special emphasis on hitherto uninvestigated Babesia spp. and Angiostrongylus vasorum, poorly investigated B. burgdorferi and A. phagocytophilum and the potentially zoonotic $D$. immitis and L. infantum.

\section{Material and methods}

\section{Samples}

All samples $(n=167)$ originated from dogs presented at the small animal clinic of the Faculty of Veterinary Medicine of Trakia University, Stara Zagora, for various clinical reasons only partly related to the infections investigated in the present study. It can be assumed that most dogs originated from the region of Stara Zagora (central-southern Bulgaria). Information about gender was available for $166 \operatorname{dogs}$ ( $n=98$ female; $n=68$ male), and the age of all 167 dogs ranged from 1 to 16 years (median 5, mean 5.5 years). There was no information available about prophylactic measures against parasites. All dogs were pet animals (no stray dogs and no dogs with a specific type of use, e.g. hunting dogs). All samples were obtained and examined with the agreement of the owners.

\section{Methods}

All samples were examined by means of $\mathrm{SNAP}^{\circledR}$ $3 \mathrm{Dx}{ }^{\circledR}$ enzyme immunoassay (EIA), A. phagocytophilum indirect immunofluorescence assay (IFA), B. canis microplate ELISA and L. infantum microplate ELISA. In total 150 of the samples (due to a shortage of material from the remaining 17 samples) were examined for the presence of circulating A.vasorum antigen and of specific antibodies against $A$. vasorum by validated ELISAs. Three samples (one with very low positive and two with borderline $L$. infantum ELISA test results and concurrent high $B$. canis ELISA results) were additionally investigated by means of L.infantum IFA to exclude potential cross-reactions with anti-B. canis antibodies, as reported for a similar L.infantum ELISA (according to Mettler et al. 2005; Wolf et al. 2014). Twenty-seven samples that reacted positively for anti-Ehrlichia antibodies within the SNAP $^{\circledR} 3 \mathrm{Dx}^{\circledR}$ EIA were additionally examined by means of $\mathrm{SNAP}^{\circledR} 4 \mathrm{Dx}^{\circledR}$ Plus EIA in order to detect potential cross-reactions of anti-E. canis antibodies with A.phagocytophilum whole-cell antigen (as present on IFA slides), as described by Harrus and Waner (2011). All tests were performed in Ludwigsburg, Germany (IDEXX Laboratories), apart from A. vasorum antigen and antibody ELISAs that were performed in Zurich, Switzerland (Institute of Parasitology, University of Zurich). Differences between prevalence rates were analysed for significance using the Chi-square test (differences were regarded as significant at a level of $p<0.05$; Bland 2000). Data was evaluated via Excel (MS). 


\section{A. phagocytophilum serology (IFA)}

Serological examinations for antibody detection were performed using A. phagocytophilum IFA (MegaScreen FLUOANAPLASMA ph., cut-off $=1: 50$, MegaCor, Hoerbranz, Austria). The test uses slides coated with A.phagocytophilum-infected cells (see also Dyachenko et al. 2012). The instructions of the manufacturer were slightly modified. Thus, the positive cut-off was set at a clear green fluorescence of the cytoplasmatic inclusion bodies (morulae) at dilutions of $\geq 1: 200$. Titres of 1:50 and 1:100 were considered borderline. The kit contents were modified by using another FITC-conjugated secondary antibody at a dilution of 1:1600 (fluorescein-labelled affinity purified antibody to dog IgG $(\mathrm{H}+\mathrm{L})$ produced in goat, Kirkegaard and Perry Laboratories/KPL) with Evans Blue (2.5 ml, Bio$\mathrm{Rad})$ as the counter stain, and Biognost ${ }^{\circledR}$ mounting medium (Bios) to cover the wells prior to microscopy. Fluorescence was analysed using an Olympus BX41 microscope equipped with an Osram Mercury Short Arc HOB ${ }^{\circledR}$ lamp (103 W/2).

\section{Leishmania serology (ELISA, IFA)}

Serological examinations for antibody detection were performed using a microplate L.infantum ELISA (Leishmania-ELISA Dog, Afosa GmbH, Dahlewitz, Germany). The reference range of the test score (TS; the test converts the colorimetric reaction through a formula into "test scores") is negative below 7 , borderline at 7-12 and positive above 12 according to Wolf et al. (2014). Test performance and evaluation in terms of test score, sensitivity, specificity as well as positive and negative predictive values as compared to IFA and another microplate ELISA are described by Wolf et al. (2014). L. infantum IFA was performed according to Wolf et al. (2014).

\section{Babesia serology (ELISA)}

Serological examinations for antibody detection were performed using a microplate $B$. canis ELISA (Babesia-ELISA Dog, Afosa GmbH, Dahlewitz, Germany; the reference range of the test score (see above) is negative $<14$, borderline at $14-19$ and positive $>19$ ). According to the test manufacturer, the sensitivity and the specificity of the B.canis ELISA compared with the indirect immunofluorescence assay (IFA) (671 sera tested using four different IFAs) are $91.6 \%$ and $95.4 \%$, respectively. In relation to a "gold standard" IFA validated at a university institute according to strict scientific criteria (287 sera), sensitivity and specificity are as high as $96.3 \%$ and $100 \%$. This ELISA is currently the only test authorised by the German Friedrich Loeffler Institute for the detection of B.canisspecific antibodies in dogs. However, it shows cross-reactivity with other Babesia spp. (such as $B$. vogeli according to Dyachenko et al. 2012) and with $B$. gibsoni and Rangelia vitalii (Pantchev et al. 2015). This whole-cell based assay can be therefore rather regarded as piroplasma-specific.

\section{Angiostrongylus vasorum serology (antigen and antibody detection; ELISAs)}

The ELISA for antigen detection with a sensitivity of $95.7 \%$ and a specificity of $94.4 \%$ was performed as previously described (Schnyder et al. 2011). For the detection of specific antibodies a sandwich ELISA using A.vasorum adult somatic antigen purified by monoclonal antibodies (mAb Av 5/5) was used, with a sensitivity of $81 \%$ and a specificity of $98.8 \%$ (Schucan et al. 2012). Test cut-offs (optical density at $405 \mathrm{~nm} / \mathrm{OD}_{405}: 0.208$ for antibodies and 0.352 for antigen) were adjusted on the basis of canine study populations in Germany (Schnyder et al. 2013a) and Poland (Schnyder et al. 2013b).

\section{E. canis, B. burgdorferi, D. immitis} (antigen detection) serology (EIA)

Serological examinations were performed using a rapid test system based on an EIA technique $\left(\mathrm{SNAP}^{\circledR} 3 \mathrm{Dx}^{\circledR}\right.$, IDEXX Laboratories, Inc., Westbrook, ME, USA) performed according to the manufacturer's directions. The in-clinic assay uses a proprietary device that provides reversible chromatographic flow of sample and automatic, sequential 
flow of wash and enzyme substrate. Positive test results are detected visually as blue-coloured spots. Two spots are impregnated respectively with a specific peptide antigen of B.burgdorferi sensu lato/ $B b s l$ (C6 peptide derived from the IR6 region within the Borrelia membrane protein VlsE; O'Connor et al. 2004) and E.canis (peptides from p30 and p30-1 outer membrane proteins; cross-reactive with Ehrlichia chaffeensis; Davoust et al. 2006; O'Connor et al. 2006). The D. immitis analyte is derived from two antibodies (one for capture and the other for detection) specific to heartworm antigens, which are primarily produced by adult females (Weil 1987; Bowman et al. 2009; Chandrashekar et al. 2010).

\section{Anaplasma serology (EIA)}

Serological examination was performed using a rapid test system based on an EIA technique (SNAP $^{\circledR} 4 \mathrm{Dx}^{\circledR}$ Plus, IDEXX Laboratories, Inc., Westbrook, ME, USA). Similar to the earliergeneration EIA devices $\left(\mathrm{SNAP}^{\circledR} 3 \mathrm{Dx}^{\circledR} / 4 \mathrm{Dx}^{\circledR}\right)$, this new in-clinic EIA detects antibodies against peptides derived from the major immunodominant p30 and p30-1 proteins of E.canis and from the VlsE protein-derived C6 peptide of B. burgdorferi, but it additionally detects antibodies to Ehrlichia ewingii (peptide derived from p28 outer surface protein family) and, compared to the $\mathrm{SNAP}^{\circledR} 3 \mathrm{Dx}^{\circledR}$ used in the present study, specific antibodies to A. phagocytophilum/Anaplasma platys (peptide from the major surface protein $\mathrm{p} 44$ /MSP2) are also detected. No cross-reaction between Anaplasma and Ehrlichia at genus level is observed within this EIA device based on utilisation of specific peptides in contrast to the whole-cell antigen present on A. phagocytophilum IFA slides (Diniz and Breitschwerdt 2012; Harrus et al. 2012). The SNAP $^{\circledR}$ $4 \mathrm{Dx}^{\circledR}$ Plus test system has been validated for dogs. The sensitivity and specificity were $93.2 \%$ and $99.2 \%$ for A.phagocytophilum, $89.2 \%$ and $99.2 \%$ for A. platys, $96.7 \%$ and $98.8 \%$ for $B b s l, 97.8 \%$ and $92.3 \%$ for E.canis, and $98.9 \%$ and $99.3 \%$ for D. immitis, according to Stillman et al. (2014). This latter study furthermore showed a cross-reactivity of E. canis antigens with anti-E. chaffeensis antibodies as proposed for the earlier test generation $\left(\mathrm{SNAP}^{\circledR} 3 \mathrm{Dx}^{\circledR}\right.$, see above).

\section{Results}

Overall positive and borderline results are shown in Table 1. One hundred and eight of 167 examined samples (64.7\%; 95\% CI: 56.9-71.9\%) showed a positive result for at least one agent. The mean age for the positive tested dogs $(n=108)$ was 5.3 years and for the dogs tested negative $(n=59) 5.8$ years.

Table 1 Occurrence of Dirofilaria immitis antigen and seroprevalence of tick-borne infections in dogs from Bulgaria $(n=167)$

\begin{tabular}{|c|c|c|c|c|}
\hline Causative organism & $\begin{array}{c}\text { Antigen (Di) or } \\
\text { antibodies } \\
\text { positive/all tested } \\
\text { dogs }\end{array}$ & $\begin{array}{c}\text { Antibodies border- } \\
\text { line/all tested dogs }\end{array}$ & Percentage rate & $\begin{array}{c}\text { 95 confidence } \\
\text { interval }\end{array}$ \\
\hline Ehrlichia canis & $35 / 167$ & na & $21 \%$ & {$[15.1-27.9 \%]$} \\
\hline Dirofilaria immitis & $27 / 167$ & na & $16.2 \%$ & {$[10.9-22.6 \%]$} \\
\hline \multirow{2}{*}{ Babesia canis } & $27 / 167$ & & $16.2 \%$ & {$[10.9-22.6 \%]$} \\
\cline { 2 - 5 } & & $7 / 167$ & $4.2 \%$ & {$[1.7-8.4 \%]$} \\
\hline Borrelia burgdorferi & $4 / 167$ & na & $2.4 \%$ & {$[0.7-6 \%]$} \\
\hline $\begin{array}{c}\text { Anaplasma } \\
\text { phagocytophilum }\end{array}$ & $77 / 167$ & $42 / 167$ & $19.2 \%$ & {$[38.4-54 \%]$} \\
\cline { 2 - 5 }
\end{tabular}

na: not applicable; Di: Dirofilaria immitis 
Table 2 Proportion of single infections $(n=56)$ considering all positive samples $(n=108 ; 51.9 \% ; 95 \%$ Cl: $42-61.6 \%)$

\begin{tabular}{|c|c|c|c|c|}
\hline & Ap (alone) & Bc (alone) & Di (alone) & Ec (alone) \\
\hline positive samples & 31 & 14 & 10 & 1 \\
\hline positive tests (total) & 108 & 108 & 108 & 108 \\
\hline percentage & $28.7 \%$ & $13.0 \%$ & $9.3 \%$ & $0.9 \%$ \\
\hline $95 \% \mathrm{Cl}$ & {$[20.4-38.2 \%]$} & {$[7.3-20.8 \%]$} & {$[4.5-16.4 \%]$} & {$[0.0-5.1 \%]$} \\
\hline
\end{tabular}

Ap: Anaplasma phagocytophilum, Bc: Babesia canis, Di: Dirofilaria immitis, Ec: Ehrlichia canis

Table 3 Proportion of dual infections $(n=43)$ considering all positives samples $(n=108 ; 39.8 \% ; 95 \%$ Cl: $30.5-49.7 \%)$

\begin{tabular}{|c|c|c|c|c|c|c|}
\hline & Ap + Ec & Ap + Di & Ap + Bc & Bc + Di & Bb + Ec & Ec +Di \\
\hline positive samples & 25 & 8 & 5 & 2 & 2 & 1 \\
\hline positive tests (total) & 108 & 108 & 108 & 108 & 108 & 108 \\
\hline percentage & $23.1 \%$ & $7.4 \%$ & $4.6 \%$ & $1.9 \%$ & $1.9 \%$ & $0.9 \%$ \\
\hline $95 \% \mathrm{Cl}$ & {$[15.6-32.2 \%]$} & {$[3.3-14.1 \%]$} & {$[1.5-10.5 \%]$} & {$[0.2-6.5 \%]$} & {$[0.2-6.5 \%]$} & {$[0.0-5.1 \%]$} \\
\hline
\end{tabular}

Ap: Anaplasma phagocytophilum, Bc: Babesia canis, Bb: Borrelia burgdorferi sensu lato, Di: Dirofilaria immitis, Ec: Ehrlichia canis

Table 4 Proportion of triple and multiple infections $(n=9)$ considering all positives samples ( $n=108 ; 8.3 \% ; 95 \% \mathrm{Cl}: 3.9-15.2 \%$ )

\begin{tabular}{|c|c|c|c|c|c|}
\hline & $\mathrm{Ap}+\mathrm{Ec}+\mathrm{Di}$ & $\mathrm{Ap}+\mathrm{Bc}+\mathrm{Di}$ & $\mathbf{A p}+\mathbf{B c}+\mathrm{Ec}$ & $\mathbf{B b}+\mathrm{Ec}+\mathrm{Bc}$ & $\mathbf{B b}+\mathrm{Ec}+\mathrm{Ap}+\mathrm{Bc}$ \\
\hline positive samples & 3 & 3 & 1 & 1 & 1 \\
\hline positive tests (total) & 108 & 108 & 108 & 108 & 108 \\
\hline percentage & $2.8 \%$ & $2.8 \%$ & $0.9 \%$ & $0.9 \%$ & $0.9 \%$ \\
\hline $95 \% \mathrm{Cl}$ & {$[0.6-7.9 \%]$} & {$[0.6-7.9 \%]$} & $0.0-5.1 \%$ & $0.0-5.1 \%$ & $0.0-5.1 \%$ \\
\hline
\end{tabular}

Ap: Anaplasma phagocytophilum, Bc: Babesia canis, Bb: Borrelia burgdorferi sensu lato, Di: Dirofilaria immitis, Ec: Ehrlichia canis

The female to male ratio in the whole population was 1.4 (98 females and 68 males), while the female to male ratio among positive and negative tested dogs was 1.8 (69 females and 39 males) and 1 (females and males each of 29$)$, respectively $(p=0.083)$.

The proportions of single, dual, triple and multiple infections are shown in Tables 2-4. Slightly more single than dual infections were encountered, but the difference was not statistically significant $(p=0.076)$. However, significantly more single or dual infections than triple or multiple infections were found $(p<0.001)$. Nine of 108 positive samples showed a multiple infection with three or four agents (Table 4).
Positive ( $\mathrm{n}=27 ; 16.2 \% ; 95 \%$ CI: $10.9-22.6 \%)$ and borderline ( $\mathrm{n}=7 ; 4.2 \%$; $95 \%$ CI: $1.7-8.4 \%)$ results for B. canis serology based on ELISA TS are shown in Table 5.

All samples tested by means of L. infantum ELISA were negative, apart from two borderline samples (TS of 9.1 and 11.2) and one positive (TS of 12.6) result. These three results were interpreted as cross-reactions based on high anti-B.canis antibody levels (samples 10, 11 and 114; Table 5), and on L. infantum IFA, since all three samples were negative in this alternative confirmatory test (antibody titre $<1: 50$ ). 
Table 5 Babesia spp. positive and borderline dogs based on test score results of the ELISA applied in the present study

\begin{tabular}{|c|c|c|c|}
\hline sample number dog & TS* of positive samples & sample number dog & TS** borderline samples \\
\hline 3 & 42 & 6 & 15.3 \\
\hline 8 & 26.2 & 12 & 17.5 \\
\hline 10 & 60.9 & 43 & 17.5 \\
\hline 11 & 54.9 & 52 & 15.9 \\
\hline 27 & 23.8 & 78 & 14.3 \\
\hline 31 & 62.8 & 145 & 18.4 \\
\hline 37 & 23.3 & 147 & 17.9 \\
\hline 40 & 24.2 & & \\
\hline 41 & 23.2 & & \\
\hline 45 & 27.4 & & \\
\hline 50 & 27.6 & & \\
\hline 57 & 26.4 & & \\
\hline 85 & 19.6 & & \\
\hline 92 & 19.6 & & \\
\hline 103 & 28.1 & & \\
\hline 108 & 19.4 & & \\
\hline 114 & 197.8 & & \\
\hline 115 & 56.9 & & \\
\hline 117 & 24.3 & & \\
\hline 133 & 23.3 & & \\
\hline 139 & 23.2 & & \\
\hline 141 & 38.3 & & \\
\hline 152 & 24.1 & & \\
\hline 155 & 19.7 & & \\
\hline 160 & 31.2 & & \\
\hline 161 & 21.8 & & \\
\hline 162 & 25.4 & & \\
\hline
\end{tabular}

* range of positive TS: 19.4-197.8; mean TS of positive samples 36.8; median TS of positive samples 25.4

** range of borderline TS: 14.3-18.4; mean TS of borderline samples 16.6; median TS of borderline samples 17

All 150 samples tested for the detection of A.vasorum circulating antigen by ELISA were negative $(0 \%, 95 \%$ CI: $0.0-2.0 \%)$, while a single sample showed a reaction slightly above the cut-off $\left(\mathrm{OD}_{405}\right.$ of 0.211$)$ in the ELISA for antibody detection (1/150; $0.7 \%$; $95 \%$ CI: $0.0-3.7 \%)$.

The antibody titre of all A.phagocytophilum-positive samples (IFA, $\mathrm{n}=77$ ) was compared to the antibody titre of Anaplasma-positive, Ehrlichianegative samples $(\mathrm{n}=47$; Table 6$)$ in order to evaluate potential cross-reactions with anti-E.canis antibodies. The A. phagocytophilum antibody titre of all Ehrlichia positive samples $(\mathrm{n}=35)$ was also analysed (Table 7). Thirty out of 35 Ehrlichia positive samples were indeed A. phagocytophilum-positive (IFA), in contrast to only five samples with a negative or borderline A. phagocytophilum antibody titre (highly significant difference; $p<0.001$ ). Furthermore, the number of samples with an A. phagocytophilum antibody titre $\geq 1: 3200$ (22/35; Table 7) was significantly higher compared to all other titres $(13 / 35 ; p<0.031)$. Additionally, there 
Table 6 Antibody titre (IFA) of all Anaplasma phagocytophilum positive samples in relation to A. phagocytophilum positive but Ehrlichia canis negative samples

\begin{tabular}{|c|c|c|c|}
\hline \multicolumn{2}{|c|}{ Anaplasma positive (total $n=77 ; \mathbf{4 6 . 1} \%)$} & \multicolumn{2}{c|}{ Anaplasma positive, Ehrlichia negative $(n=47 *)$} \\
\hline antibody titre & number of samples & number of samples & significance $(p<0.05)$ \\
\hline $1: 200$ & 10 & 8 & 0.616 \\
\hline $1: 400$ & 16 & 13 & 0.536 \\
\hline $1: 800$ & 7 & 7 & 0.481 \\
\hline $1: 1600$ & 12 & 9 & 0.057 \\
\hline $1: 3200$ & 18 & 9 & $<0.001$ \\
\hline
\end{tabular}

* $28.1 \%(95 \% \mathrm{Cl}: 21.5-35.6 \%)$

Table7 A. phagocytophilum antibody titre (IFA) of all E. canis positive samples $(n=35)$

\begin{tabular}{|c|c|}
\hline Anaplasma titre & $\begin{array}{c}\text { E. canis } \\
\text { positive samples (n) }\end{array}$ \\
\hline$<1: 50$ (negative) & 3 \\
\hline $1: 50$ (borderline) & 1 \\
\hline $1: 100$ (borderline) & 1 \\
\hline $1: 200$ & 2 \\
\hline $1: 400$ & 3 \\
\hline $1: 1600$ & 3 \\
\hline $1: 3200$ & 9 \\
\hline$>1: 3200$ & 13 \\
\hline
\end{tabular}

were 30 A.phagocytophilum-borderline samples (18\%; 95\% CI: $12.5-24.6 \% ; \mathrm{n}=17$ at $1: 50$ and $\mathrm{n}=13$ at $1: 100)$ with a concurrent Ehrlichia-negative result, in contrast to only two A. phagocytophilum-borderline samples with an Ehrlichia-positive result ( $1.2 \% ; 95 \% \mathrm{CI}: 0.1-4.3 \%)$. This difference was statistically highly significant $(\mathrm{p}<0.001)$.

Twenty-seven of 35 Ehrlichia-positive samples were additionally examined by means of SNAP ${ }^{\circledR}$ $4 \mathrm{Dx}{ }^{\circledR}$ Plus EIA. Of these, 25 had a positive $A$. phagocytophilum antibody titre (one sample was negative and one had a borderline result of 1:50), but only three of them (antibody titres of 1:1600, 1:3200 and $>1: 3200)$ were confirmed positive by the specific peptide-based assay (12\%; 95\% CI: $2.5-31.2 \%$ ).

\section{Discussion}

High prevalence rates were documented for antiB. canis antibodies, D. immitis antigen ( $16.2 \%$ each), anti-E.canis (21\%) and anti-A.phagocytophilum antibodies (46.1\%), while B. burgdorferi seroprevalence was low (2.4\%). The high proportion of positive samples for at least one agent (108 of 167 tested samples; 64.7\%; 95\% CI: 56.9-71.9\%) and for dual infections $(39.8 \% ; 43 / 108)$ underlines the importance of CVBDs in pet dogs in centralsouthern Bulgaria. Even multiple infections with up to four different organisms were detected.

The high $E$. canis seroprevalence confirmed the results of previous studies from that country (Tsachev et al. 2006a, b). Although co-infections with $E$. canis and $H$. canis have also been reported from Bulgaria (Tsachev et al. 2008a), it was not possible to establish data for dual infection of this type in the present study as no serological assay was available for $H$. canis.

The mean age of positive tested dogs in the present study ( $n=108 ; 5.3$ years) was comparable to those found for positive dogs in other studies on CVBDs (Anaplasma spp., E. canis, B. burgdorferi, D. immitis), for example 6.3 years (Volgina et al. 2013) or 5 years (Villleneuve et al. 2011). While in the study of Volgina et al. (2013) the age of negative tested dogs was lower (2.6 years), this was not observed for the present study ( $n=59 ; 5.8$ years). 
In another study from Spain, even higher seropositivity for E.canis and B. burgdorferi was observed in younger dogs ( $<1$ year) compared to adult dogs, while no differences in the rates recorded for Anaplasma spp., D. immitis or L. infantum were identified (Miró et al. 2013). Overall, different studies also failed to find any association between gender and CVBDs analysed (e.g. Villleneuve et al. 2011; Miró et al. 2013).

A. phagocytophilum was the most common single infection encountered, followed by B.canis and D. immitis. The most common dual infection was A. phagocytophilum/E. canis $(\mathrm{n}=25)$, which was significantly more prevalent than the next most frequent combination of A.phagocytophilum/D. immitis $(\mathrm{n}=8 ; \mathrm{p}=0.001)$. A more in-depth discussion of this commonest co-infection is therefore considered advisable. There are various possible explanations for this result. It can be hypothesised that this represents infection by or exposure to A. platys and not A. phagocytophilum, because from the serological point of view both are cross-reactive, regardless of the test used (whole cell- or peptide-based; Gaunt et al. 2010; Dyachenko et al. 2012). In fact, a potential co-infection with $A$. platys and E.canis is even more probable considering that they use the same tick vector ( $R$. sanguineus), and that, based on previous studies, this latter tick appears to be a common ectoparasite of dogs in Bulgaria (Kirkova et al. 2013). However, there are so far no documented reports of $A$. platys occurring in Bulgaria, and no EDTA blood was available for species-specific PCR tests or PCR with subsequent sequencing for reliable molecular differentiation in the present study, as described previously by Dyachenko et al. (2012). Another possible explanation for the above-mentioned common co-infection is a serological cross-reaction of $E$. canis-infected dogs with the A.phagocytophilum analyte on IFA slides. An assumed disadvantage of A.phagocytophilum whole cell-based IFA compared to tests utilising specific peptides (e.g. SNAP ${ }^{\circledR} 4 \mathrm{Dx}^{\circledR}$; Chandrashekar et al. 2010) is indeed a possible cross-reactivity with anti-E.canis antibodies. The strength of this cross-reactivity increases with the duration of the E.canis infection and the anti-E.canis antibody titre (Harrus et al. 2012). For example, in dogs infected experimentally with $E$. canis, no cross-reactivity with A. phagocytophilum antigens (IFA) was observed in the acute phase. However, cross-reactive antibodies were first detected on day 55 post infection (p.i.), were then found in all dogs by day 150 p.i. and even increased in two dogs until 283 days p.i. (Waner et al. 1998; Harrus and Waner 2011). This could be due to variation of antibody patterns during E. canis infection, with initial production of antibodies against low molecular weight proteins and, subsequently, during the chronic phase of infection, against larger proteins above $36 \mathrm{kDa}$ (the latter representing cross-reactive common antigens between the two species). Waner et al. (1998) proposed furthermore that the appearance of IgG antibodies against A. phagocytophilum antigens after $E$. canis infection may even represent an indicator of long-term persistence of infection and replication of $E$. canis in the canine host. In the present study, a set $(\mathrm{n}=27)$ of $E$. canis-positive samples was tested using the latest generation of the abovementioned peptide-based assay ( $\operatorname{SNAP}^{\circledR} 4 \mathrm{Dx}^{\circledR} \mathrm{Plus}$ ) that shows no cross-reactivity between the genera Anaplasma and Ehrlichia. Out of 25 E. canis-positive samples with an A.phagocytophilum IFA titre above the test cut-off, only three confirmed positive within the specific peptide-based assay. It was also evident that especially samples with a high antiAnaplasma antibody titre ( $\geq 1: 3200)$ were Ehrlichia-positive, indicating samples with very high Ehrlichia antibody levels and/or long-term, chronic $E$. canis infections. It is therefore difficult to predict the real seroprevalence of $A$. phagocytophilum with the method (IFA) used in the present study. A possible way of estimating it would be to consider only the Anaplasma IFA-positive but Ehrlichia-negative samples $(n=47)$; furthermore to assume that only $12 \%$ (i.e. $\mathrm{n}=4$ ) of Anaplasma/Ehrlichia co-infected samples are true positives (proportion of samples confirmed by peptide-based EIA). With this approach, the estimated Anaplasma seroprevalence 
would be $30.5 \%$ (51/176; $95 \%$ CI: $23.7-38.1 \%)$. At any rate, if the borderline results are added to this (30 of 32 were indeed Ehrlichia-negative), then again a comparable high Anaplasma seroprevalence of $48.5 \%$ (95\% CI: $40.7-56.3 \%$ ) would be achieved. Nevertheless, at least three samples (Ehrlichia-positive in $\mathrm{SNAP}^{\circledR} 3 \mathrm{Dx}^{\circledR}$ and additionally Anaplasma-positive in $\mathrm{SNAP}^{\circledR} 4 \mathrm{Dx}^{\circledR}$ Plus) can be regarded as a co-infection or at least co-exposure to both organisms. Interestingly, in experimental canine models (A. platys and E. canis) it was shown that subsequent acute $E$. canis infection can trigger the antibody response of a previous Anaplasma infection (Gaunt et al. 2010).

Surprisingly, all four Borrelia-positive samples were detected as mixed infections with at least E. canis. This indicates another intriguing co-infection that points on the one hand to exposure of dogs to at least two different tick vectors, I. ricinus and $R$. sanguineus. On the other hand, it is known that mixed infection with Lyme borreliae and rickettsias in the form of A.phagocytophilum increases the potential of a dog to develop clinical signs, in contrast to infections with a single agent (Beall et al. 2008). Nevertheless, Borrelia seroprevalence in dogs in Bulgaria is relatively low compared with Germany (9.7\%; Krupka et al. 2007) but comparable with Poland, for example, (3.8\%; Krämer et al. 2014) and even higher than in France (1.1\%; Pantchev et al. 2009), all results obtained with a comparable C6-based EIA.

A relatively high infection rate was found for D. immitis antigen detection compared with previous investigations (Geogieva et al. 2001; Kirkova et al. 2008). One reason could be that previous studies relied mainly on microfilariae detection, and it is well known that up to $70 \%$ of heartworm infections can be occult and are only detected by antigen methods (Pantchev et al. 2011). Mixed infection with D. immitis and D. repens as described by Pantchev et al. (2011) for dogs exported from Bulgaria to Germany could not be detected in the present study as no microfilariae-based tests (e.g. Knott's, filtration or PCR tests) were performed. To date, no serological test is available for $D$. repens, and the heartworm antigen test used in the present study does not react with $D$. repens (Pantchev et al. 2011). Moreover, cross-reaction as shown for some D. immitis antigen tests with sera of A.vasorum infected dogs (Schnyder and Deplazes 2012) could be excluded. In the present study, no A.vasorum circulating antigen was detected. All positive reactions for D. immitis antigen in the present study can therefore be regarded as specific serological reactions. A single dog was A.vasorum antibody-positive, which could be interpreted as a false positive reaction. In areas with expected low prevalence like Bulgaria, both A. vasorum ELISAs should be performed because seropositivity in both tests has a considerable high positive predictive value (Schnyder et al. 2013a). These results do not necessarily exclude the presence of A. vasorum in Bulgaria (no A. vasorum cases were reported so far), as the sampling number was restricted (indicated also by the large confidence intervals) and as the dogs originated from a restricted area around Stara Zagora, which is located in the central-southern part of the country. Considering the large number of recent reports of A.vasorum in several European countries, including Poland (Schnyder et al. 2013b) or northern Greece (Papazahariadou et al. 2007), bordering to Bulgaria, the detection of cases of A.vasorum has actually to be expected.

The situation with L. infantum was similar. One low positive sample and two borderline results were interpreted as cross-reactions with Babesia as shown, for example, by Mettler et al. (2005) as these samples also had high anti-Babesia antibody levels. Leishmania IFA for these three samples was negative, a constellation reported to occur with comparable ELISA and IFA tests (Mettler et al. 2005). This corroborates previous data of a seroepidemiological study (using IFA) that was carried out with 220 healthy pet dogs from 11 different Bulgarian regions (5 in the south, including 20 samples from Stara Zagora and 20 samples from the Plovdiv area, and 6 northern regions; Tsachev et al. 2007b). All samples in this latter study also tested negative for 
Leishmania antibodies. The results of these studies contrast with human data from Bulgaria where, despite a low annual incidence of 0.06 per 100,000 , most cases of Leishmania were reported from the southern part of the country, including the area of Stara Zagora (Harizanov et al. 2013). As high percentages of D. immitis infections in dogs were detected in the present study, the application of repellents with efficacy against insects, particularly mosquitoes and sand flies, seems not to be the reason for the negative Leishmania results. One explanation could be that $P$. papatasi and $P$. sergenti, which are the main sand fly species in Bulgaria, have a higher tropism towards humans than towards pet dogs, in contrast to $P$. neglectus, which is the main sand fly species transmitting $L$. infantum encountered in Greece (Léger et al. 1988; Garifallou et al. 1989; Killick-Kendrick and Killick-Kendrick 1999; Tsachev et al. 2007b). Additionally, many asymptomatic dogs in endemic areas mount predominantly cellular immunity with a negative to low specific antibody titre and may therefore be missed by serological methods (Baneth et al. 2008). For example, of 73 clinically healthy hunting dogs in neighbouring Greece, $12.3 \%$ tested positive by serology, whereas $63 \%$ tested positive by PCR (Leontides et al. 2002). The latter authors concluded that most of the dogs residing in areas where leishmaniosis is endemic become infected but often remain seronegative. Serological screening of the general canine population in these areas may therefore result in an underestimation of the true prevalence.

Data in the present study represent the first serological evidence of canine babesiosis in Bulgaria. It can be concluded that exposure to Babesia spp. seems to be common in dogs in Bulgaria. The most probable species involved in canine infections in this country is $B$. vogeli due to suitable vector availability (R. sanguineus) and its common finding on dogs, though, the presence of $B$. canis cannot be excluded. Further studies are necessary (as in Croatia, for example; Beck et al. 2009) to reliably differentiate serologically cross-reactive Babesia species by means of species-specific real time PCRs or PCRs with subsequent sequencing or RFLP analysis in order to exactly determine the species involved. In regard to $B$. gibsoni, there is also speculation about non-vector-borne direct dog-to-dog transmission via bite wounds, saliva or ingested blood (fighting dogs; Irwin 2009), but so far no B. gibsoni has been described in Bulgaria.

There is one limitation to this serological survey as a positive antibody result is not necessarily equivalent to the existence of the pathogen in the canine or vector population of a particular geographic region; it is only evidence of prior exposure to the corresponding pathogen at some point and some location in the dog's history. With respect to the latter limitation, a more differentiated view needs to be taken of the detection of antibodies against $\mathrm{C} 6$ peptide of B. burgdorferi. The SNAP ${ }^{\circledR} 4 \mathrm{Dx}^{\circledR}$ Plus test detected infections with Borrelia at the earliest on day 35 or day 49 p.i., depending on the individual dog (Wagner et al. 2012). On the other hand, antibodies to C6 have been detected in the late stages of infection (>12 months) with a $\mathrm{C} 6$ detecting device (Levy et al. 2008; Wagner et al. 2012) and have been found to decrease significantly after specific treatment, so that at least for Borrelia the detection of $\mathrm{C} 6$ peptide might represent a more or less robust marker of infection.

In conclusion, this study represents an overview of the prevalence of important canine, but also zoonotic, pathogens in a canine population in Bulgaria. Our data underline the importance of CVBDs and especially co-infections which could influence the clinical outcome in dogs. Veterinarians in this country should be aware that these major canine vectorborne pathogens may occur in their practice area. Therefore, clinicians should include these diseases in their differential diagnosis and recommend the use of repellents along with prophylactic measures to prevent disease transmission by arthropod vectors.

\section{Ethical standards}

All investigations comply with the current laws of the countries in which they were performed. 


\section{Competing interests}

N. Pantchev and M. Globokar are employed by IDEXX Laboratories. There is no commercial con- flict of interest as the information generated here is solely for scientific dissemination. The authors declare that they have no competing interests.

\section{References}

Baneth G, Koutinas AF, Solano-Gallego L, Bourdeau P, Ferrer L (2008): Canine leishmaniosis - new concepts and insights on an expanding zoonosis: part one. Trends Parasitol 24(7):324-30

Beck R, Vojta L, Mrljak V, Marinculić A, Beck A, Zivicnjak T, Cacci ${ }^{\circledR}$ SM (2009) Diversity of Babesia and Theileria species in symptomatic and asymptomatic dogs in Croatia. Int J Parasitol 39(7):843-8

Beall MJ, Chandrashekar R, Eberts MD, Cyr KE, Diniz PP, Mainville C, Hegarty BC, Crawford JM, Breitschwerdt EB (2008) Serological and molecular prevalence of Borrelia burgdorferi, Anaplasma phagocytophilum, and Ehrlichia species in dogs from Minnesota. Vector Borne Zoonotic Dis 8:455-464

Bland JM (2000) An introduction to medical statistics. $3^{\text {th }}$ edition, Oxford university press, 230

Bowman D, Little SE, Lorentzen L, Shields J, Sullivan MP, Carlin EP (2009) Prevalence and geographic distribution of Dirofilaria immitis, Borrelia burgdorferi, Ehrlichia canis, and Anaplasma phagocytophilum in dogs in the United States: results of a national clinic-based serologic survey. Vet Parasitol 160(1-2):138-48

Chandrashekar R, Mainville CA, Beall MJ, O'Connor T, Eberts MD, Alleman AR, Gaunt SD, Breitschwerdt EB (2010) Performance of a commercially available in-clinic ELISA for the detection of antibodies against Anaplasma phagocytophilum, Ehrlichia canis, and Borrelia burgdorferi and Dirofilaria immitis antigen in dogs. Am J Vet Res 71(12):1443-50

Davoust B, Bourry O, Gomez J, Lafay L, Casali F, Leroy E, Parzy D (2006) Surveys on seroprevalence of canine monocytic ehrlichiosis among dogs living in the Ivory Coast and Gabon and evaluation of a quick commercial test kit dotELISA. Ann N Y Acad Sci 1078:464-9

Diniz PP, Breitschwerdt EB (2012) Anaplasma phagocytophilum infection (canine granulocytotropic anaplasmosis). In: Infectious diseases of the dog and cat. $4^{\text {th }}$ edition, edited by Greene CE., Elsevier, 244-254

Dyachenko V, Pantchev N, Balzer HJ, Meyersen A, Straubinger RK (2012) First case of Anaplasma platys infection in a dog from Croatia. Parasit Vectors 5:49
Garifallou A, Hadziandoniou M, Schnur LF, Yuval B, Warburg A, Jacobson RL, Pateraki E, Patrikoussis M, Schlein Y, Sérié C (1989) Epidemiology of human and canine leishmaniasis on the island of Zakinthos. In: Hart DT (ed.): Leishmaniasis. The current status and new strategies for control. NATO ASI Series/A, Life Sciences Vol. 163, Plenum Press, New York, 1011-1015

Georgieva D, Kirkova Z, Ivanov A (2001) A study on the incidence and diagnosistics of dirofilariosis (heartworm disease) in carnivores. Bulg J Vet Med 4(4):231-236

Gaunt S, Beall M, Stillman B, Lorentzen L, Diniz P, Chandrashekar R, Breitschwerdt E (2010) Experimental infection and co-infection of dogs with Anaplasma platys and Ehrlichia canis: hematologic, serologic and molecular findings. Parasit Vectors 3(1):33

Harizanov R, Rainova I, Tzvetkova N, Kaftandjiev I, Bikov I, Mikov O (2013) Geographical distribution and epidemiological characteristics of visceral leishmaniasis in Bulgaria, 1988 to 2012. Euro Surveill 18(29):20531

Harrus S, Waner T (2011) Diagnosis of canine monocytotropic ehrlichiosis (Ehrlichia canis): an overview. Vet J 187(3):292-6

Harrus S, Waner T, Neer TM (2012) Ehrlichia canis infection. In: Infectious diseases of the dog and cat. $4^{\text {th }}$ edition; edited by Greene CE. Elsevier; 227-238

Irwin PJ (2009) Canine babesiosis: from molecular taxonomy to control. Parasit Vectors 2 Suppl 1:S4

Ivanov IN, Mitkova N, Reye AL, Hübschen JM, VatchevaDobrevska RS, Dobreva EG, Kantardjiev TV, Muller CP (2011) Detection of new Francisella-like tick endosymbionts in Hyalomma spp. and Rhipicephalus spp. (Acari: Ixodidae) from Bulgaria. Appl Environ Microbiol 77(15):5562-5

Killick-Kendrick R, Killick-Kendrick M (1999) Biology of sand fly vectors of Mediterranean canine leishmaniasis. Proceedings of the International Canine Leishmaniasis Forum, Barcelona, Spain: 26-31

Kirkova Z, Ivanov A, Georgieva D, Prelesov P (2008) An update on dirofilariosis in dogs and wild canines in Bulgaria. Sbornik dokladi ot nauchnata konferentsiya: Traditsii i s'vremenhost v'v veterinarnata meditsina:247-252, in Bulgarian 
Kirkova Z, Iliev P, Vesser M, Knaus M (2013) Survey on ectoparasites of dogs (Canis familiaris) in Bulgaria. Conference Proceedings, 12th International Symposium of Ectoparasites in Pets, Munich:16

Krämer F, Schaper R, Schunack B, Połozowski A, Piekarska J, Szwedko A, Jodies R, Kowalska D, Schüpbach D, Pantchev N (2014) Serological detection of Anaplasma phagocytophilum, Borrelia burgdorferi sensu lato and Ehrlichia canis antibodies and Dirofilaria immitis antigen in a countrywide survey in dogs in Poland. Parasitol Res 113(9):3229-39

Krupka I, Pantchev N, Weise M, Straubinger RK (2007) [Tick-transmitted, bacterial infections in dogs: Seroprevalences of Anaplasma phagocytophilum, Borrelia burgdorferi sensu lato and Ehrlichia canis in Germany]. Durch Zecken übertragbare bakterielle Infektionen bei Hunden: Seroprävalenzen von Anaplasma phagocytophilum, Borrelia burgdorferi sensu lato und Ehrlichia canis in Deutschland. Praktischer Tierarzt 10(88):776-87, in German

Léger N, Gramiccia M, Gradoni L, Madulo-Leblond G, Pesson B, Ferté H, Boulanger N, Killick-Kendrick R, KillickKendrick M (1988) Isolation and typing of Leishmania infantum from Phlebotomus neglectus on the island of Corfu, Greece. Trans R Soc Trop Med Hyg 82(3):419-20

Leontides LS, Saridomichelakis MN, Billinis C, Kontos V, Koutinas AF, Galatos AD, Mylonakis ME (2002) A crosssectional study of Leishmania spp. infection in clinically healthy dogs with polymerase chain reaction and serology in Greece. Vet Parasitol. 109(1-2):19-27

Levy SA, O’Connor TP, Hanscom JL, Shields P, Lorentzen L, Dimarco AA (2008) Quantitative measurement of C6 antibody following antibiotic treatment of Borrelia burgdorferi antibody-positive nonclinical dogs. Clin Vaccine Immunol 15(1):115-9

Mettler M, Grimm F, Capelli G, Camp H, Deplazes P (2005) Evaluation of enzyme-linked immunosorbent assays, an immunofluorescent-antibody test, and two rapid tests (immunochromatographic-dipstick and gel tests) for serological diagnosis of symptomatic and asymptomatic Leishmania infections in dogs. J Clin Microbiol 43(11):5515-9

Mikov O (2011) Distribution of mosquitoes potentially transmitting West Nile virus in Bulgaria. $9^{\text {th }}$ national congress on clinical microbiology and infection, Bulgarian association of microbiologists, Plovdiv, 28-30 April, 2011:45-46, in Bulgarian

Mikov O, Harizanov R (2012) Distribution of the phlebotomine sandflies in Bulgaria and their importance as vectors of parasitic and viral diseases. $10^{\text {th }}$ National Congress of Clinical Microbiology and Infections of the Bulgarian Association of Microbiologists, April 26-28, 2012, Plovdiv, Bulgaria. Book of abstracts: $47-48$
Miró G, Montoya A, Roura X, Gálvez R, Sainz A (2013) Seropositivity rates for agents of canine vector-borne diseases in Spain: a multicentre study. Parasit Vectors 6:117

O’Connor TP, Esty KJ, Hanscom JL, Shields P, Philipp MT (2004) Dogs vaccinated with common Lyme disease vaccines do not respond to IR6, the conserved immunodominant region of the VlsE surface protein of Borrelia burgdorferi. Clin Diagn Lab Immunol 11(3):458-62

O'Connor TP, Hanscom JL, Hegarty BC, Groat RG, Breitschwerdt EB (2006) Comparison of an indirect immunofluorescence assay, western blot analysis, and a commercially available ELISA for detection of Ehrlichia canis antibodies in canine sera. Am J Vet Res 67(2):206-10

Pantchev N, Schaper R, Limousin S, Norden N, Weise M, Lorentzen L (2009) Occurrence of Dirofilaria immitis and tick-borne infections caused by Anaplasma phagocytophilum, Borrelia burgdorferi sensu lato and Ehrlichia canis in domestic dogs in France: results of a countrywide serologic survey. Parasitol Res 105 Suppl 1:S101-14

Pantchev N, Etzold M, Daugschies A, Dyachenko V (2011) Diagnosis of imported canine filarial infections in Germany 2008-2010. Parasitol Res 109 Suppl 1:S61-76

Pantchev N, Pluta S, Huisinga E, Natter S, Scheufelen M, Globokar Vrhovec M, Schweinitz A, Hampel H, Straubinger RK (2015) Tick-borne diseases (borreliosis, anaplasmosis, babesiosis) in German and Austrian Dogs: status quo and review of distribution, transmission, clinical findings, diagnostics and prophylaxis. Parasitol Res 114 Suppl 1:S13-48

Papazahariadou M, Founta A, Papadopoulos E, Chliounakis S, Antoniadou-Sotiriadou K, Theodorides Y (2007) Gastrointestinal parasites of shepherd and hunting dogs in the Serres Prefecture, Northern Greece. Vet Parasitol 148:170-173.

Popova T, Kamenov S (1998) Erythematous form of Lyme in dog. Veterinary Medicine 3-4:244-246

Schnyder M, Tanner I, Webster P, Barutzki D, Deplazes P (2011) An ELISA for sensitive and specific detection of circulating antigen of Angiostrongylus vasorum in serum samples of naturally and experimentally infected dogs. Vet Parasitol 179:152-158

Schnyder M, Deplazes P (2012) Cross-reactions of sera from dogs infected with Angiostrongylus vasorum in commercially available Dirofilaria immitis test kits. Parasit Vectors 5:258

Schnyder M, Schaper R, Bilbrough G, Morgan ER, Deplazes $P$ (2013a) Seroepidemiological survey for canine angiostrongylosis in dogs from Germany and the UK using combined detection of Angiostrongylus vasorum antigen and specific antibodies. Parasitology 140(11):1442-50 
Schnyder M, Schaper R, Pantchev N, Kowalska D, Szwedko A, Deplazes P (2013b) Serological detection of circulating Angiostrongylus vasorum antigen- and parasite-specific antibodies in dogs from Poland. Parasitol Res 112 Suppl 1:109-17.

Schucan A, Schnyder M, Tanner I, Barutzki D, Traversa D, Deplazes P (2012) Detection of specific antibodies in dogs infected with Angiostrongylus vasorum. Vet Parasitol 185:216-224

Stillman BA, Monn M, Liu J, Thatcher B, Foster P, Andrews B, Little S, Eberts M, Breitschwerdt EB, Beall MJ, Chandrashekar R (2014) Performance of a commercially available in-clinic ELISA for detection of antibodies against Anaplasma phagocytophilum, Anaplasma platys, Borrelia burgdorferi, Ehrlichia canis, and Ehrlichia ewingii and Dirofilaria immitis antigen in dogs. J Am Vet Med Assoc 245(1):80-6

Tsachev I (2006) Detection of antibodies reactive with $E h r$ lichia canis in a kennel in Bulgaria. Turk J Vet Anim Sci 30:425-426

Tsachev I, Kontos V, Zarkov I, Krastev S (2006a) Survey of antibodies reactive with Ehrlichia canis among dogs in South Bulgaria. Rev Med Vet 157(10):481-485

Tsachev I, Papadagjannakis E, Kontos V, Zarkov I, Petrov V, Pelagic V (2006b) Seroprevalence of Ehrlichia canis infection among privately-owned dogs in northern Bulgaria. J Hell Vet Med Soc 57(3):212-216

Tsachev I, Simeonov R, Petrov V (2007a) Infections with Ehrlichia canis and Borrelia burgdorferi in a dog. Veterinarski glasnik 61(3-4): 201-210

Tsachev I, Papadajonakis M, Kontos V, Ivanov A, Chakarova B, Stojanchev K, Peshev R (2007b) Seroepidemiology of Leishmania infantum exposure among healthy dogs in Bulgaria. Turk J Vet Anim Sci 31(1):73-4

Tsachev I, Ivanov A, Dinev I, Simeonova G, Kanakov D (2008a) Clinical Ehrlichia canis and Hepatozoon canis coinfection in a dog in Bulgaria. Rev Med Vet 159(2):68-73

Tsachev I, Petrov V, Fleming K, Brown C (2008b) First detected case of Anaplasma phagocytophilum in a dog in Bulgaria. Revue de Médecine Vétérinaire 159 (11):562-564

Tsachev I (2009) Exotic zoonoses among dogs in Bulgaria. DSc Thesis:315, in Bulgarian
Tsachev I, Petrov V (2009) A clinical case of asymptomatic pancytopaenia in a dog. Scientific works, C series, LV (2), Veterinary Medicine, UASVM, Bucharest:29-33

Tsachev I, Kyriazis I, Karagouni E, Boutsini S, Dotsika E (2010): First report of canine visceral leishmaniasis in Bulgaria. Turk J Vet Anim Sci 34(5):465-469

Tsachev I, Dimov I (2012) Seroprevalence of Borrelia burgdorferi in dogs in Bulgaria. 2nd International Epizootiology Days, book of abstracts, Belgrade: 175

Tsachev I, Gundasheva D, Papadagjannakis E, Kontos V, Denev S (2013) Haematological profiles in canine monocytic ehrlichiosis: a retrospective study of 31 spontaneous cases in Greece. Rev Med Vet 164(6): 327-330

Villeneuve A, Goring J, Marcotte L, Overvelde S (2011) Seroprevalence of Borrelia burgdorferi, Anaplasma phagocytophilum, Ehrlichia canis, and Dirofilaria immitis among dogs in Canada. Can Vet J 52(5):527-30

Volgina NS, Romashov BV, Romashova NB, Shtannikov AV (2013) Prevalence of borreliosis, anaplasmosis, ehrlichiosis and Dirofilaria immitis in dogs and vectors in Voronezh Reserve (Russia). Comp Immunol Microbiol Infect Dis 36(6):567-74

Wagner B, Freer H, Rollins A, Garcia-Tapia D, Erb HN, Earnhart C, Marconi R, Meeus P (2012) Antibodies to Borrelia burgdorferi OspA, OspC, OspF, and C6 antigens as markers for early and late infection in dogs. Clin Vaccine Immunol 19(4):527-35

Waner T, Strenger C, Keysary A, Harrus S (1998) Kinetics of serologic cross-reactions between Ehrlichia canis and the Ehrlichia phagocytophila genogroups in experimental E. canis infection in dogs. Vet Immunol Immunopathol $66(3-4): 237-43$

Weil GJ (1987) Dirofilaria immitis: identification and partial characterization of parasite antigens in the serum of infected dogs. Exp Parasitol 64:244-251

Wolf D, Failing K, Taubert A, Pantchev N (2014) Serological diagnosis of canine leishmaniosis: comparison of three commercially available tests. Parasitol Res 113(5):1997-2002 